EESTI NSV TEADUSTE AKADEEMIA TOIMETISED. 29. KÖIDE FOOSIKA * MATEMAATIKA. 1980, NR. 3

ИЗВЕСТИЯ АКАДЕМИИ НАУК ЭСТОНСКОИ ССР. ТОМ 29 ФИЗИКА * МАТЕМАТИКА. 1980, № 3

\title{
РАССЕЯНИЕ АКУСТИЧЕСКОГО ИМПУЛЬСА НА СПЛОШНОМ УПРУГОМ ЦИЛИНДРЕ
}

\author{
(Представил Н. Алумяэ)
}

Излагаются результаты вычисления частотной и временной зависимостей поля давления. Проводится детальный анализ структуры временной зависимости, описываются ее основные компоненты и информационные признаки. Предлагается процедура отыскания физических параметров упругого цилиндра по рассчитанной временной зависимости.

\section{1. Постановка задачи и метод решения}

Пусть в безграничной жидкости поконтся упругий цилиндр бесконечной протяженности. На него падает плоский акустический импульс давления, фронт которого параллелен продольной оси цилиндра, а зависимость изменения давления за фронтом волны может быть задана произвольно. Требуется рассчитать поле акустического давления в жидкости, вызванное падающим импульсом, связать свойства этого поля со свойствами упругого цилиндра и дать алгоритм нахождения физических параметров упругого цилиндра по рассчитанным параметрам рассеянного поля.

Будем полагать, что движение жидкости описывается волновым уравнением, а движение упругого цилиндра - уравнениями линейной теории упругости. Примем, что поперечное сечение цилиндра имеет форму круга. Отсчет времени начнем с момента, когда падающий импульс коснется поверхности цилиндра. Поставленная выше физическая задача математически сводится к решению трех волновых уравнений, одно из которых отвечает движению жидкости, а два других - движению упругого цилиндра, связанных между собой посредством условий контакта на поверхности цилиндра, а именно - непрерывностью радиальных перемещений и напряжений и отсутствием тангенциальных напряжений. Задача решается при нулевых начальных условиях: полагается, что все искомые функцин и их первые производные по времени в начальный момент равны нулю. Из физнческих соображений следует, что искомые функции ограничены в областях, где они определены, а цавление в жидкости, помимо этого, удовлетворяет условию излучения Зоммерфельда.

Для решения задачи применяется интегральное преобразование Фурье по времени ( $F$-преобразование). В таком случае в пространстве $F$-преобразования волновые уравнения переходят в уравнения Гельмгольца. Используется метод разделения переменных по радиальной и угловой координатам и выписывается решение в виде бесконечного ряда, коэффициенты которого определяются из $F$-преобразованных ус- 
ловий контакта. При каждом значении индекса суммирования получается система из трех алгебраических уравнений для отыскания неизвестных коэффициентов. Решением этой системы завершается построение точного аналитического решения задачи в пространстве $F$-преобразования. Обратное преобразование Фурье выполняется численно с использованием экономной процедуры численного интегрирования Ромберга. Со всеми деталями метода решения и алгоритма вычисления можно ознакомиться в $\left[{ }^{1}\right]$.

Введем следующие обозначения: $R, \theta-$ радиальная и угловая координаты, $T-$ время, $a-$ наружный радиус цилиндра; $E, v, \varrho_{1}-$ модуль упругости, коэффициент Пуассона и плотность материала цилиндра; $c_{1}, c_{2}$ - скорости распространения продольной и поперечной волн в линейной теории упругости, $p_{s}$ - давление в рассеянном упругим цилиндром поле, $\mathrm{Q}_{0}, c_{0}$ - плотность и скорость звука в жидкости.

Удобно ввести безразмерные переменные

$$
r=R / a, \quad t=c_{0} T / a .
$$

Падающий импульс (волновой пакет) для конкретности примем в виде

$$
\begin{gathered}
p_{i}=p_{*} \sin \left(x_{0} \delta\right)\left[H(\delta)-H\left(\delta-\delta_{0}\right)\right], \\
\delta=t+(r \cos \theta-1),
\end{gathered}
$$

где $p_{i}$ - давление в падающем импульсе, $p_{*}$ - постоянная, имеющая размерность давления, $x_{0}-$ частота заполнения, $\tau_{0}=2 \pi / x_{0}-$ период частоты заполнения, $\delta_{0}$ - длительность волнового пакета.

Давление в рассеянном поле $p_{s}$ нормируем на геометрический множитель

$$
p=p_{s} / m, \quad m=p_{*}[r(2 \cos \gamma / \cos \alpha-1 / r)]^{-1 / 2},
$$

причем угол наблюдения $\theta$ и угол.падения $\alpha$ связаны уравнениями

$$
\sin \alpha=r \sin \gamma, \quad \theta f \gamma-2 \alpha=0 .
$$

Давление в некоторой фиксированной точке $p(\tau)$ измеряется с момента, когда рассеянное цилиндром поле придет в точку наблюдения: $\tau=t-(1-2 \cos \alpha+r \cos \gamma) . \quad F$-преобразование давления $p(\tau)$ обозначим через $p^{F}(x)$. В дальнейшем ради краткости будем называть $p(\tau)$ временной зависимостью, а $p^{F}(x)$ - частотной зависимостью.

\section{2. Численњые результаты}

Вычисление акустического давления в воде, рассеянного сплошным стальным цилиндром, проводилось 'в точке наблюдения $r=10^{4}, \theta=0$ при $x_{0}=9,84, \delta_{0}=3 \tau_{0}$ и

$$
\begin{array}{ll}
\mathrm{Q}_{0}=1,0 \mathrm{r} / \mathrm{cm}^{3}, & c_{0}=1493 \mathrm{M} / \mathrm{c}, \\
\mathrm{Q}_{1}=7,7 \mathrm{r} / \mathrm{cm}^{3}, & c_{1}=5960 \mathrm{M} / \mathrm{c}, \quad c_{2}=3240 \mathrm{M} / \mathrm{c} .
\end{array}
$$

На рис. 1 показана зависимость модуля $\left|p^{F}(x)\right|$ частотной характеристики, вычисленная на ЭВМ ЕС-1033, с шагом по $x$, равным 10/256. Временная зависимость рассчитывалась при частоте $x_{0}=9,84$, при которой $\left|p^{F}(x)\right|$ имеет локальный максимум. Обратное преобразование Фурье выполнялось численно; интегрирование по частоте $x$ проводилось от 0 до 100 . 

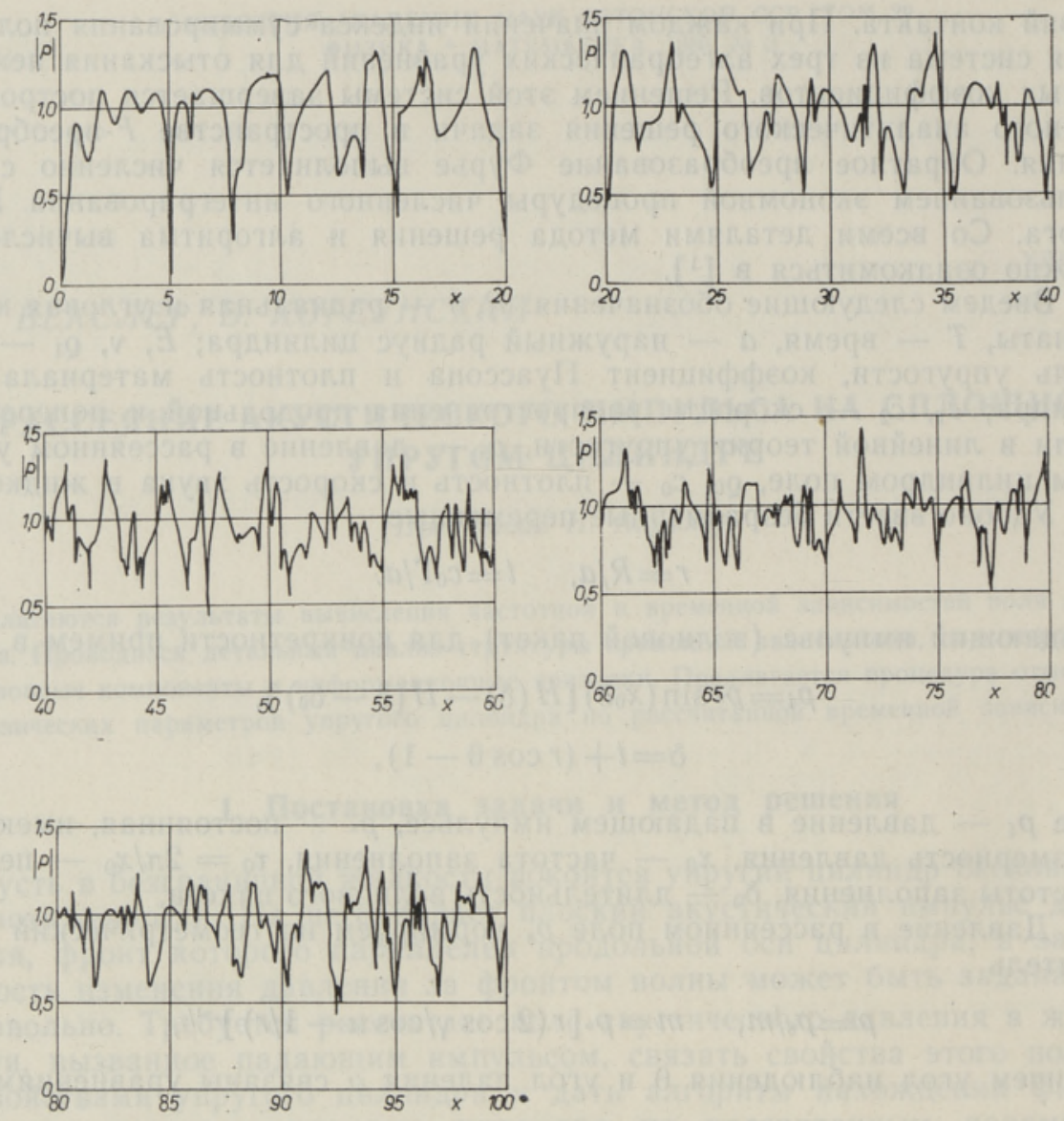

Рис. 1.

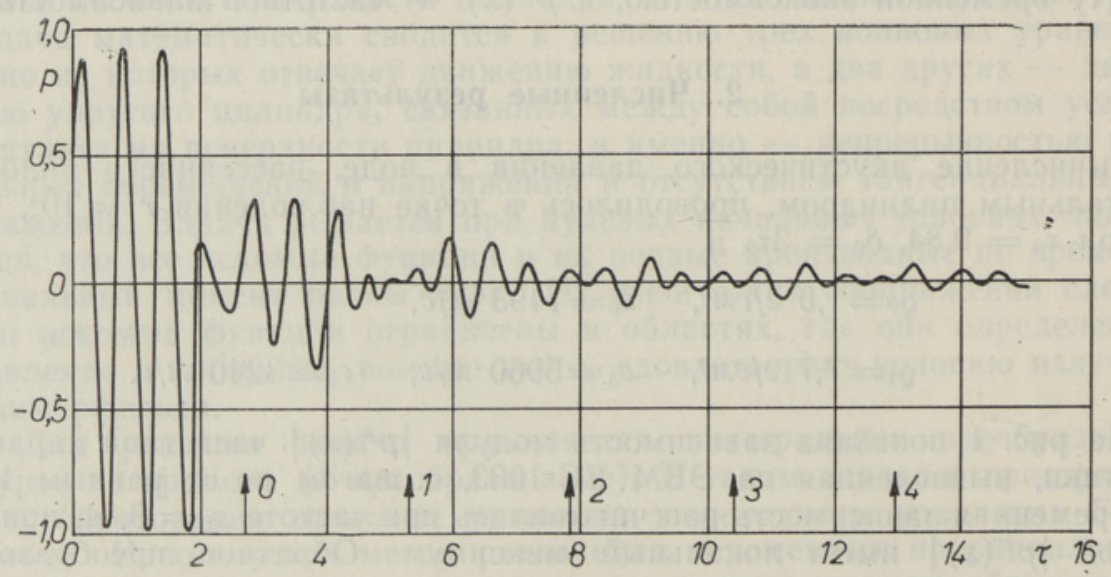

Рис. 2. 
На рис. 2. представлен график временной зависимости. Видно, что один падающий волновой пакет (три периода синусоиды в пакете) вызывает последовательность пакетов в рассеянном поле. Рассеянное поле формируется за счет отражения, переотражения, переизлучения и дифракции. Первый пакет на рис. 2 обусловлен процессами отражения и переотражения, а последующие, в основном, - переизлучением.

\section{3. Анализ численных результатов}

Рассмотрим детально структуру кривой временной зависимости. Прежде всего отметим, что эту кривую можно разделить на пакеты. Среднюю величину модуля амплитуды максимумов и минимумов в пределах одного пакета условимся называть амплитудой пакета. Первый пакет имеет амплитуду порядка единицы, а амплитуды последующих пакетов затухают по экспоненте. Так, например, амплитуда нулевого переизлученного пакета, простирающегося по $\tau$ от 2,64 до 4,62 , имеет порядок трех десятых, а четвертого - порядок пяти сотых.

Отражение и пе ре отражени е. Рассмотрим подробно первый пакет. Он образуется, в основном, за счет отражения от наружной поверхности упругого цилиндра и переотражения (с последующим выходом к точке наблюдения) от его тыльной поверхности. При малых значениях $\tau$ (вплоть до момента прихода волнового пакета, отраженного от задней поверхности цилиндра) амплитуда временной зависимости может быть получена посредством умножения ординат временной зависимости от «акустически жесткого» неподвижного цилиндра на коэффициент отражения $v$ для случая плоской границы раздела жидкой и упругой сред при нормальном падении (при параметрах, определенных формулой (5), он равен 0,937$)$. В приведенном рассуждении используется т. н. принцип изолированного элемента $\left[{ }^{2}\right]$, согласно которому от каждой точки поверхности гладкого упругого тела волновой пакет отражается как от точки касательной плоскости. Любопытно отметить, что при выводе принципа изолированного элемента использовалась коротковолновая (высокочастотная) асимптотика решения волновых уравнений. В обсуждаемом примере, хотя длина волны $\lambda=0,639$ составляет более половины радиуса цилиндра, принцип изолированного элемента «работает».

Рассеянное цилиндром поле рассчитано на луче $\theta=0$. При нормальном падении коэффициент отражения от плоской границы раздела жидкой и упругой сред совпадает с соответствующим коэффициентом для двух жидких полупространств (поперечные волны не возбуждаются), а в случае наклонного падения - отличается от него. При цилиндрической границе раздела коэффициенты отражения и прохождения достаточно сложны и зависят от того, с какой стороны (выпуклой или вогнутой) волновой пакет падает на границу. В случае цилиндрической границы раздела в данную точку наблюдения «светит» не одна отражающая точка, а вся дуга на цилиндрической поверхности. В принципе, для коротких волн (при $\lambda \ll 1)$ известно, как рассчитать отраженный и переотраженный пакеты в случае упругого цилиндрического отражателя $\left[{ }^{3}\right]$; однако формулы для коэффициентов отражения и прохождения являются довольно сложными, имеется много типов лучей, много различных коэффициентов и, что самое неприятное, приходится считать эйконалы всех лучей с учетом многократных переотражений. С ростом частоты эти формулы переходят в формулы коэффициентов 
отражения и прохождения для плоской границы раздела, которые и используются нами, даже при малых частотах.

Для оценки вклада поперечных волн во временную зависимость было рассмотрено рассеяние волнового пакета в случае модельной задачи, когда поперечные волны не возникают. С этой целью в формуле (5) было принято $c_{2}=0$ и проведен расчет для «жидкого стального» цилиндра. Из рис. 3 видно, что до. прихода волнового пакета, отраженного от задней поверхности, результаты расчета временной зависимости для упругого и жидкого цилиндров практически совпадают, что свидетельствует о том, что вклад во внешнее акустическое поле поперечных волн, образовавшихся за счет трансформации продольных волн на границе раздела, невелик и им можно пренебречь.

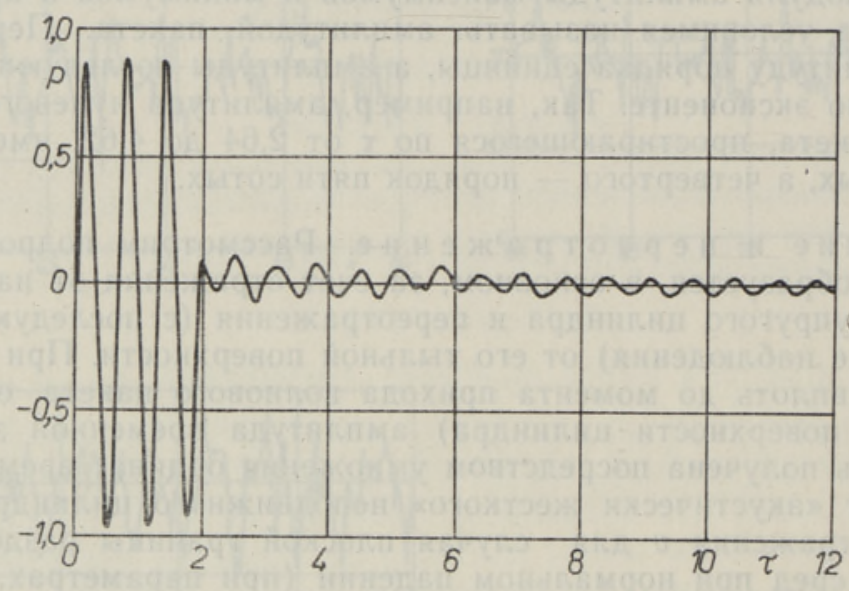

Рис. 3 .

\begin{tabular}{l|c|r|r|r}
\hline \multirow{2}{*}{ Точка } & \multirow{2}{*}{$\tau$} & \multicolumn{3}{|c}{ Цилиндр } \\
\cline { 3 - 5 } & & упругий & жидкий & жесткий \\
\hline$I_{\max }$ & 0,155 & 0,88 & 0,87 & 0,88 \\
$I_{\min }$ & 0,470 & $-0,97$ & $-0,98$ & $-0,97$ \\
$I_{\max }$ & 0,790 & 0,89 & 0,89 & 0,88 \\
II $_{\min }$ & 1,105 & $-0,96$ & $-0,96$ & $-1,01$ \\
III $_{\max }$ & 1,425 & 0,91 & 0,88 & 0,92 \\
III $_{\min }$ & 1,740 & $-0,96$ & $-0,93$ & $-1,00$
\end{tabular}

В таблице приведены времена прихода и величины амплитуд максимумов и минимумов первого пакета временной зависимости, а также, для сравнения, амплитуды соответствующих величин для жидкого цилиндра и «акустически жесткого» неподвижного цилиндра (последние умножены на коэффициент отражения в случае плоской границы раздела жидких полупространств при нормальном падении).

Данные таблицы указывают на то, что начиная с $\tau=1,0$, т. е. с момента прихода волнового пакета, отраженного от задней поверхности, результаты в столбцах существенно различаются, как и следовало ожидать. Действительно, при $\tau>1$ начинают сказываться как кривизна отражающей поверхности (на отраженный и переотраженный волновые пакеты), так и вклад поперечных волн. Заметим, что в последнем столбце учитывается только процесс отражения от наружной поверхности. Если из временной зависимости, рассчитанной для упругого ци- 
линдра (рис. 2), вычесть временную зависимость, рассчитанную для «акустически жесткого» цилиндра, предварительно умноженную на $v$, то разница будет отчетливо давать волновой пакет, переотраженный от задней поверхности цилиндра. В частности, для рассматриваемого примера видно, что такой волновой пакет запаздывает по сравнению с зеркально отраженным на $\tau=1,0$, что соответствует его пути вдоль диаметра цилиндра (согласно представлениям геометрической оптики).

Длинный «хвост» на рис. 3 обусловлен процессом переотражения в жидком цилиндре. Интенсивность переотраженных волновых пакетов падает с ростом числа переотражений, что проявляется в спадании амплитуды переотраженного волнового пакета от величины порядка $5 \%$ при $\tau=2-4$ до величины порядка $2 \%$ при $\tau=10-12$. Если бы удалось отделить процесс переотражения от процесса переизлучения, то подобный «хвост» наблюдался бы и в случае упругого цилиндра.

П е р е и з у ч ен и е. Сопоставление графиков на рис. 2 и 3 позволяет легко отличить упругий цилиндр от жидкого по наличию на рис. 2 бо́льших по амплитуде и периодически повторяющихся по $\tau$ переизлученных волновых пакетов. Как известно, при падении волнового пакета на упругий цилиндр в последнем возбуждаются поверхностные волны. Будем называть эти волны волнами типа Рэлея на цилиндрической поверхности. Они возникают на критическом угле $\theta=\theta_{3} \approx$ $\approx \arcsin \left(k_{3} / k\right)$, когда проекция фазовой скорости падающего волнового пакета на направление касательной к окружности контакта равна скорости звука в жидкости, обегают цилиндр по часовой стрелке и против нее, непрерывно излучая в жидкость под тем же критическим углом. При нулевом угле наблюдения $\theta=0$ волновые пакеты, вызванные этими обегающими цилиндр волнами, приходят в точку наблюдения одновременно и суммируются. Если затухание поверхностной волны типа Рэлея невелико (как это имеет место в рассматриваемом случае), то она до своего затухания совершает несколько полных оборотов, а в точку наблюдения приходят излученные этой волной волновые пакеты. Будем называть всю их последовательность переизлученными волновыми пакетами. Первый из них, порожденный волной типа Рэлея, которая не успевает совершить ни одного полного оборота вокруг поверхности цилиндра, назовем нулевым переизлученным пакетом, второй из них - первым переизлученным и т. д.

Как показано в $\left[{ }^{4}\right]$, в случае наклонного падения плоской акустической волны на плоскую границу раздела идеальной сжимаемой жидкости и упругого полупространства, когда угол падения имеет значение $\theta_{3}$, максимальная доля энергии падающей волны переходит в волну типа Рэлея. По-видимому, подобное имеет место и для упругого цилиндра (во всяком случае это наблюдается при выбранных в задаче параметрах).

Используя характерные интервалы между последовательными переизлученными волновыми пакетами, можно легко рассчитать относительную фазовую скорость поверхностной волны типа Рэлея $c_{3}{ }^{p h}=2,42 c_{0}$ и критический угол возбуждения (излучения) $\theta_{3}=0,427$ рад $=24,4^{\circ}$.

Ниже приведены примерные моменты прихода повторных переизлученных волновых пакетов:

\begin{tabular}{l|c|c|c|c|c} 
Число оборотов & 0 & 1 & 2 & 3 & 4 \\
\hline Момент прихода & 2,68 & 5,28 & 7,88 & 10,48 & 13,08
\end{tabular}


Эти времена помечены на рис. 2 стрелками. В той части кривой, где переизлученные волновые пакеты отчетливо разделяются, совпадение времен, рассчитанных по фазовой скорости и вычисленных на ЭВМ, хорошее; там же, где переизлученные волновые пакеты переходят друг в друга (накладываются друг на друга), моменты их прихода определяются с некоторой погрешностью. Такая погрешность может возникать и вследствие того, что рассчитанная зависимость включает в себя излучения всех переотраженных и переизлученных упругим цилиндром волн (т. е. волн типа Стонели, типа Франца, продольных и поперечных волн типа шепчущей галереи). Мы же полагали, что в этой сумме преобладает один тип волн - поверхностная волна типа Рэлея, а вклад остальных волн невелик.

В предположении, что затухание поверхностной волны типа Рэлея носит экспоненциальный характер, определим коэффициент затухания $\eta$ по средней амплитуде в нулевом и четвертом переизлученных волновых пакетах. Эти средние амплитуды составляют 0,2975 и 0,0450. Таким образом, за безразмерное время $8 \pi$ (четыре оборота вокруг цилиндра) поверхностная волна уменьшается в 6,62 раза, что дает коэффициент затухания $\eta=0,0752$.

Располагая частотной зависимостью (см. рис. 1), можно определить групповую скорость переизлученного волнового пакета $c_{3}{ }^{g r}=$ $=\Delta x c_{0}$, где $\Delta x-$ расстояние между характерными минимумами (либо максимумами) на частотной зависимости. Принятая частота заполнения $x_{0}$ лежит в интервале от 7,89 до 10,31 между последовательными минимумами на стационарной кривой и, следовательно, $\Delta x=2,42$. Таким образом, при выбранных параметрах контактирующих сред и заданной длине волны фазовая и групповая скорости оказались равными $c_{3}{ }^{p h}=$ $=c_{3}{ }^{g r}$. Это возможно в том случае, когда $c_{3}{ }^{g r}$ не зависит от частоты, и тогда волновой пакет распространяется без дисперсии.

Волна типа Рэлея, действительно, мало подвержена дисперсии. Из теории интегрального преобразования Зоммерфельда-Ватсона известно, что поведение поверхностной волны полностью определяется координатами полюса на комплексной плоскости интегрального преобразования. В частности, вещественная часть координаты полюса определяет величину фазовой скорости

$$
c_{3}^{p h}=\left[\operatorname{Re} v_{3}\right]^{-1} c_{0} .
$$

Как следует из результатов расчета координат полюсов $v_{3}$ для случая алюминиевого цилиндра в воде [ $\left.{ }^{5}\right]$, величина $\operatorname{Re} v_{3}$ изменяется от 0,48 до 0,51 при изменении частоты $x$ от 25 до 100, что указывает на слабую зависимость фазовой скорости от частоты. Если фазовая скорость мало меняется по частоте, то групповая скорость примерно равна фазовой, что хорошо согласуется с нашим результатом и характером частотной зависимости, изображенной на рис. 1 .

По нашему расчету (см. рис. 2), нулевой переизлученный волновой пакет приходит в фазе с отраженным, а согласно результату эксперимента, проведенного для алюминиевого цилиндра в воде $\left[{ }^{6}\right]$, - в противофазе. Мы дополнительно исследовали этот вопрос. К сожалению, нам не удалось найти ни одной статьи, где была бы рассчитана временная зависимость для упругого цилиндра. Поэтому мы провели расчет рассеянного поля в случае явно несимметричной временной зависимости в падающем волновом пакете

$$
p_{i}=\left(p_{*} / 2\right)\left[\sin x_{0} \psi-\cos 2 x_{0} \psi\right]\left[H(\psi)-H\left(\psi-\delta_{0}\right)\right], \quad \psi=\delta+\pi / 6 x_{0} .
$$



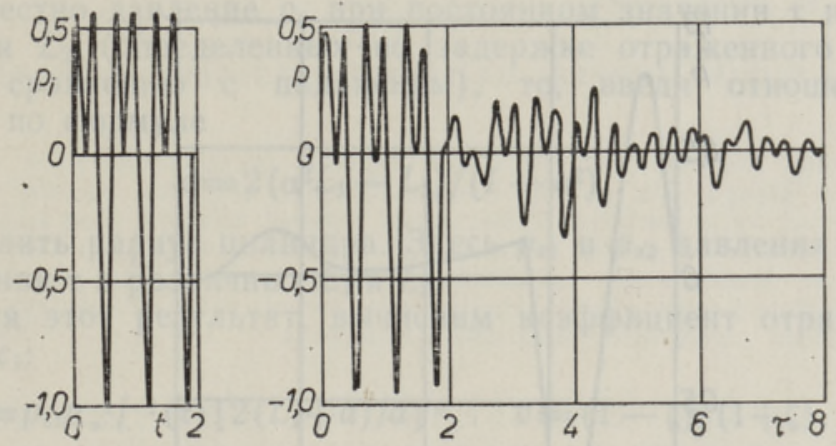

Рис. 4.

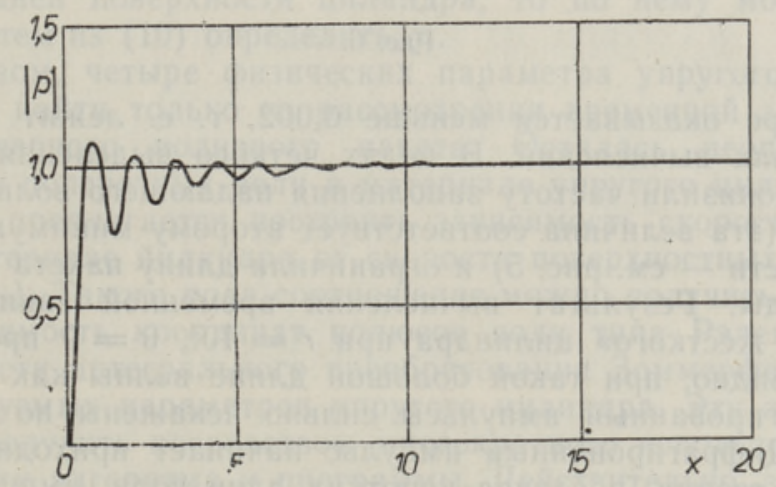

Рис. 5.

На рис. 4 показаны результаты такого расчета. Слева - падающий волновой пакет, справа - временная зависимость в рассеянном поле. Все остальные параметры те же, что и прежде. Как видно, нулевой переизлученный волновой пакет приходит в фазе с отраженным.

Д и фр а кц и я. Как известно, дифракция в узком смысле (огибание препятствия) на упругом теле происходит почти так же, как на «акустически жестком». Действительно, как это следует из $\left[{ }^{7}\right]$, полюса волн типа Франца, порождающие дифракционные волны, в случае упругого цилиндра близки к таковым в случае «акустически жесткого» цилиндра. Координаты полюсов типа Франца на упругом цилиндре для больших длин волн $(\lambda \sim 1)$ получаются посредством разложения их в ряд Тейлора по частоте, нулевой член которого - координата полюса типа Франца на «акустически жестком» цилиндре. При больших длинах волн первый и последующие члены этого ряда дают небольшую поправку к нулевому члену. С уменьшением длины волны влияние этих поправок сходит на нет, и при $\lambda \ll 1$ координаты полюсов волн типа Франца для упругого и «акустически жесткого» цилиндров практически совпадают. Фазовая скорость волн типа Франца чуть меньше скорости звука в окружающей цилиндр жидкости и в расчетах ее можно принять равной этой скорости. Их амплитуда при длинных волнах может составить несколько процентов от амплитуды зеркально отраженного импульса. При выбранной нами частоте заполнения падающего волнового пакета $x_{0}=9,84$ амплитуда волны типа Франца на «акустически жест- 


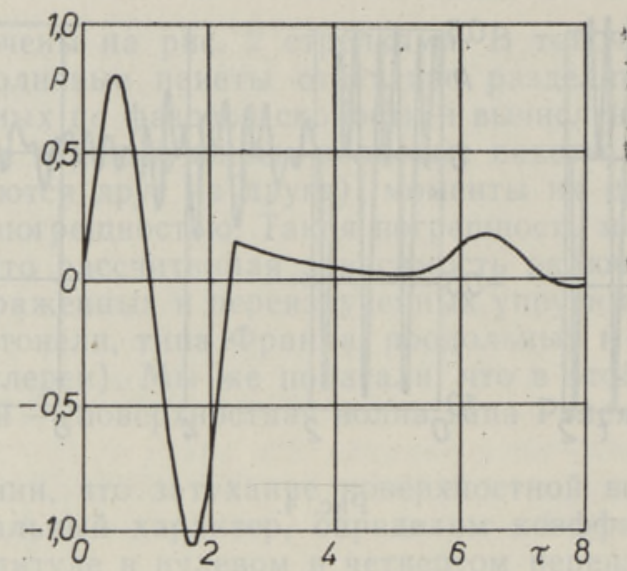

Рис. 6.

ком» цилиндре оказывается меньше 0,002 , т. е. лежит ниже порога точности наших вычислений. В целях четкого выделения волны типа Франца мы понизили частоту заполнения падающего волнового пакета до $x_{0}=2,64$ (эта величина соответствует второму минимуму на частотной зависимости - см. рис. 5) и ограничили длину пакета одним периодом синусоиды. Результат вычисления временной зависимости для «акустически жесткого» цилиндра при $r=10^{4}, \theta=0$ представлен на рис. 6. Как видно, при такой большой длине волны как отраженный, так и дифрагированный импульсы сильно искажены по сравнению с падающим. Дифрагированный импульс начинает приходить в момент $\tau=5,26$ (по теории, в случае коротких длин волн, импульс запаздывает на $\tau=\pi+2)$. В приведенном примере, хотя $\lambda=2,38(\lambda>2 !)$, лучевая картина геометрической оптики для путей дифрагированных волн все еще «работает».

\section{4. Информационные признаки временной зависимости и инверсная задача}

Зная структуру временной зависимости, найдем в ней информационные признаки и используем их для решения инверсной задачи - определения параметров упругого цилиндра по временной зависимости.

Предположим, что на упругий цилиндр падает плоский волновой пакет, фронт которого параллелен продольной оси цилиндра. Пусть поперечное сечение цилиндра - круг. При такой априорной информации посредством вычисления амплитуды и времени прихода некоторой характерной точки временной зависимости (напр., первого минимума в первом пакете) в две различные фиксированные точки наблюдения на луче $\theta=0$ можно найти радиус цилиндра. Согласно изложенному выше, в той части временной зависимости, которая описывает только процесс отражения, давление в рассеянном поле может быть приближенно представлено в виде

$$
p_{s}=p * v[2(L+a) / a]^{-1 / 2} f(\tau),
$$

где $L$ - расстояние от источника (он же приемник) до наружной поверхности упругого цилиндра, $f(\tau)$ - заданная функция режима источника, 
Если известно давление $p_{s}$ при постоянном значении $\tau$ и двух различных $L_{1}$ и $L_{2}$ (определенных по задержке отраженного волнового пакета по сравнению с падающим), то, введя отношение $\alpha^{2}=$ $=\left(p_{s 1} / p_{s 2}\right)^{2}$ по формуле

$$
a=2\left(\alpha^{2} L_{1}-L_{2}\right) /\left(1-\alpha^{2}\right),
$$

легко вычислить радиус цилиндра. Здесь $p_{s 1}$ и $p_{s 2}$ давления при постоянном значении $\tau$ и различных $L_{1}$ и $L_{2}$.

Используя этот результат, вычислим коэффициент отражения $v$ и импеданс $Q_{1} c_{1}$ :

$$
\begin{gathered}
v=p_{s 1} p_{*}^{-1} f^{-1}(\tau)\left[2\left(L_{1}+a\right) / a\right]^{1 / 2}, \quad v=(1-\zeta) /(1+\zeta), \\
\zeta=\left(\varrho_{0} c_{0}\right) /\left(\varrho_{1} c_{1}\right), \quad \varrho_{1} c_{1}=\varrho_{0} c_{0}(1+v) /(1-v) .
\end{gathered}
$$

Если теперь использовать время прихода $T_{1}$ волнового пакета, отраженного от задней поверхности цилиндра, то по нему можно найти $c_{1}=4 a / T_{1}$, а затем из (10) определить $Q_{1}$.

Таким образом, четыре физических параметра упругого цилиндра из пяти можно найти только из рассмотрения временной зависимости в диапазоне первого волнового пакета. Осталась неопределенной только скорость поперечных волн в материале упругого цилиндра. Для ее нахождения предлагается построить зависимость скорости поперечных волн в материале цилиндра от скорости поверхностных волн типа Рэлея $c_{2}=c_{2}\left(c_{3}\right)$. Такого рода соотношение можно получить, например, исследуя зависимость координат полюсов волн типа Рэлея на комплексной плоскости интегрального преобразования Зоммерфельда-Ватсона от варьируемых параметров упругого цилиндра. Эту зависимость можно также получить посредством многократного использования разработанных нами алгоритма и программы. Действительно, зависимость $c_{3}=c_{3}\left(c_{2}\right)$ определяется по нашим расчетам точно и надежно. Так что изменяя $c_{2}$ в интересующем диапазоне, можем определить $c_{3}=c_{3}\left(c_{2}\right)$, а затем инвертировать зависимость, используя ее как $c_{2}=c_{2}\left(c_{3}\right)$.

\section{ЛИТЕРАТУРА}

1. Векслер Н. Д., Рассеяние импульсов на упругих цилиндрах, Таллин, «Валгус», 1980.

2. Гел ьчин ский Б. Я., Докл. АН СССР, 118, № 3, 458-460 (1958)

3. B ri11, D., Ober a 11, H., J. Acoust. Soc. Amer., 50, № 3 (Part 2), 921-939 (1971).

4. B unney, R. E., Goodman, R. R., J. Acoust. Soc. Amer., 53, № 6, 1653-1662 (1973)

5. Dickey, J. W., Uber a 11, H., J. Acoust. Soc. Amer., 66, № 1, 275-283 (1979).

6. D r a g o n e t te, L. R., J. Acoust. Soc. Amer., 65, № 6, 1570-1572 (1979).

7. Frisk, G. V., Dickey, J. W., Ubera11, H., J. Acoust. Soc. Amer., 58, № 5, 996-1008 (1975).

Институт кибернетики Академии наук Эстонской ССР
Поступила в редакцию 6/III 1980 


\section{N. VEKSLER, V. KORSUNSKI}

\section{AKUSTILISE IMPULSI HAJUMINE ELASTSELT SILINDRILT}

Hüdrolokatsioonist on teada, et uuritavalt objektilt peegeldunud laine kuju sarnaneb sondeeriva laine kujuga. See on õige ka regulaarsete objektide, näiteks silindri puhui. Artiklis on uuritud vette asetatud homogeenset elastset silindrit ning näidatud teoreetiliselt, et kõik vaadeldavad efektid tekivad silindri vibratsioonist, mida põhjustab silindrile langev sondeeriv impulss. On analüüsitud tekkinud laine kuju muutumist sōltuvalt ajast ning kirjeldatud tema põhilisi komponente, samuti esitatud uuritava objekti mõotmete ja füüsikaliste parameetrite määramise metoodika.

\section{N. VEKSLER, V. KORSUNSKI}

\section{SCATTERING OF SOUND BY ELASTIC CYLINDER}

It is well known that an incident pulse upon a target embedded into water causes a scattered field formed by a sequence of pulses. These pulses (an echo-signal) are caused by reflection, multiple reflection, and radiation. In this paper the time-dependence of the echo-signals on a homogeneous elastic cylinder in water is analysed and the main components of it are described. It is shown that the reflected pulse (the first packet of the echo-signal) could be determined using the principle of the isolated element $\left[^{2}\right]$. The influence of the multiple reflected pulses and of the transient waves was investigated using the solution of a model problem - the problem of the «liquid steel cylinder». This problem corresponds to the case of the usual steel cylinder when the velocity of the shear waves is equal to zero.

The second and the following packets in the echo-signal form are due to the superposition of multiple reflected and radiated waves. It is assumed that the Rayleigh wave is the most essential one and the influence of the other waves could be neglected. The relative phase velocity of the Rayleigh wave, the critical angle and the attenuation coefficient are calculated. Using the frequency relation, the group velocity of the Rayleigh wave is calculated. The phase and group velocities are equal in this case.

Generally speaking, the phase velocity of the Rayleigh wave does not depend essentially on the frequency $\left[{ }^{5}\right]$ and therefore the phase and group velocities must be quite close. This agrees well with our approximate solution.

Calculations show that the Rayleigh wave (the second packet in the echo-signal) has the same phase as the reflected pulse, whereas according to the experiment $\left.{ }^{6}\right]$ it must have the opposite phase.

The informative parameters in the echo-signal are distinguished and the procedure for solving the inverse problem is analysed. The solution of the inverse problem permils to determine the parameters of an elastic circular cylinder. 